\title{
Nodes Deployment Scheme of Heterogeneous Wireless Sensor Network Based on Organic Small Molecule Model
}

\author{
Juwei Zhang ${ }^{1,2,3}$, Yu Wang ${ }^{1,2}$, Yachuang Liu ${ }^{1}$, and Qiangyi Li ${ }^{1,2}$ \\ ${ }^{1}$ Henan University of Science and Technology, Henan Luoyang 471023, China \\ ${ }^{2}$ Power Electronics Device and System Engineering Lab of Henan, Henan Luoyang 471023, China \\ ${ }^{3}$ Xian Jiaotong University, Shanxi Xian 710049, China \\ Corresponding author, e-mail:
}

\begin{abstract}
For nodes deployment of heterogeneous sensor network, based on different probability sensing models of heterogeneous nodes, refer to organic small molecule model, 'class-molecule' sensing model of heterogeneous nodes is proposed. Combined with DSTT data fusion model, the changes of network coverage ratio after using the new sensing model and data fusion algorithm is studied. According to the research results, the node-deployment strategy of heterogeneous sensor networks based on organic small molecule model (NHOS) is proposed. Sensor network simulation model is established using MATLAB software, the results show the effectiveness of the algorithm, the coverage of network and detection efficiency of nodes are improved, the lifetime of network is prolonged, energy consumption and the number of deployment nodes is reduced, and the scope of perceiving is expanded. As a result, NHOS can improve the detection performance of the network.
\end{abstract}

Keywords: node deployment scheme, heterogeneous wireless sensor network, organic small molecule model

Copyright ( 2015 Institute of Advanced Engineering and Science. All rights reserved.

\section{Introduction}

With the development of wireless sensor technology and manufacturing microelectronic technology, the wireless sensor network consist of a large number of micro sensor nodes which own perception, calculation and communication ability is applied to the military or civilian areas, such as environmental monitoring, industrial control, battlefield surveillance, detection of highrisk environment, biological medicine, intelligent household and health monitoring, etc [1].

Coverage problem is a basic problem in wireless sensor networks deployment [2], under the condition of the sensor node energy, perception, communication and computing ability limited, using a certain strategy of software and hardware, which can ensure the coverage area and coverage time, realize effective awareness and monitoring, is an important indicator of network perception service quality, and also is a hot problem in sensor network research [3].

In many applications, there are usually a variety of monitoring objects in the monitoring area. Such as, the sensors nodes need to monitor water temperature, salinity, ph value, etc in water environmental monitoring, and the sensors nodes need to monitor a variety of chemical diffusion in factory pollution early warning [4].

Since the hardware cost of current sensor node is higher, in multi-object monitoring applications, each node assembly a variety of different types of sensors, these nodes are heterogeneous. When the node energy is limited, the more sensors are assembled on a node; the life of the node is shorter. Two important problems should be considered in multiple object monitoring network coverage, namely how to use less cost of network to obtain the ideal network coverage performance, and how to weigh the importance of network monitoring of different objects according to different objects [5].

Heterogeneous characteristics of heterogeneous wireless sensor network give expression to three aspects including node heterogeneity, link heterogeneity and network protocol heterogeneity [6-10]. Node heterogeneity includes perception ability, calculation ability, 
communication ability, energy, etc. And the communication ability, perception ability, and energy make the biggest influence on coverage. The coverage problem research in the existing literature about randomly deployment of heterogeneous wireless sensor network is less.

A wireless sensor network coverage optimization algorithm is presented to perceive the radius of the heterogeneous, which can effectively improve the heterogeneous network coverage in [4]; Based on integer vector planning, A multi-objective coverage algorithm is proposed in [5], which can effectively solve the problem of multiple target monitoring. Both of the two perception model which used in [4] and [5] is relatively simple, and can not meet the demand of the true condition.

A routing protocol based evolutionary algorithm is proposed for the heterogeneous cluster node of WSN in literature [11], Which can effectively reduce the errors of cluster nodes handling data combination and separation, prolong the survival time of network, but did not give the algorithm of non-cluster node. An efficient dynamic clustering strategy (EDCS) which can effectively solve the selection problem of the multi-level heterogeneous network cluster node, effectively improve the performance of network and prolong the survival time of network is put forward in literature [12]; however, EDCS did not give a specific node deployment algorithm.

A new idea is proposed by increasing heterogeneous nodes to prolong the survival of WSN in literature [13], but the heterogeneous node perception problem was not considered. To solve the sensing radius of heterogeneous network node deployment problem, Expandingvirtual force algorithm (EX-VFA) algorithm is put forward in [14], but the connectivity and data fusion problem is little considered in EX-VFA.

For heterogeneous Sensor network nodes coverage blind area problem, a node deployment coverage algorithm based on organic small molecules model NHOS (Nodes Deployment Strategy of Heterogeneous Sensor Networks-based on Organic Small Molecule Model, NHOS) is proposed in this paper. Based on the organic small molecule structure model, heterogeneous nodes detection model is established. In order to improve the network coverage, extend the life of the network and achieve the optimal deployment of sensor nodes to monitored area, A data fusion model was proposed, combining with the DSmT data fusion and decision rules. Randomly deployment mobile nodes will move according to the principle of "virtual potential field". The effectiveness of NHOS is verified by simulation experiments, which can effectively reduce the number of deployment node and the node energy consumption, improve the efficiency of network coverage, prolong the network lifetime, expand the detection range, and improve the detection performance of the network.

\section{Mathematical Model and Assumption}

We assume that the monitoring area $\mathrm{H}$ by rectangular, $\mathrm{N}$ kinds of heterogeneous perception ability of wireless sensor nodes randomly distributed within the rectangle $\mathrm{H}$, and also assume that the wireless sensor network (WSN) has the following properties:

(1) Heterogeneous perception ability sensor. Sensors have different perception ability, the perception of radius is different, and the node model perception is probability perception model. The position of sensor node $\mathrm{i}$ is $\left(x_{i}, y_{i}\right)$, perception distance is $r_{i}$ and perceived probability is $P_{i}$ which meet the function $P_{i}\left(r_{i}\right)$. The typical perception probability model [13] is as follows:

$$
P\left(S_{i}, Q\right)=\left\{\begin{array}{cc}
0, & r+r_{e} \leq d_{i p}, \\
\frac{E_{i r}}{E_{i}} e^{\left(-\lambda \alpha^{\beta}+\alpha \delta\right)}, & r-r_{e} \leq d_{i p} \leq r+r_{e}, \\
1, & r-r_{e} \geq d_{i p} .
\end{array}\right.
$$

Where $P\left(S_{i}, Q\right)$ is the perception probability of the sensor node $S_{i}$ to target node $Q, d_{i p}$ is Euclidean distance between sensor node $S_{i}$ and the target node $\mathrm{Q}, r_{e}\left(r_{e}<r\right)$ is a uncertainty perception measure of the sensors, $E_{i}$ is the initial energy of sensor nodes, $E_{i r}$ is the remaining energy, $\alpha=d_{i p}-\left(r-r_{e}\right), \lambda$ and $\beta$ is the perception of sensor nodes within the scope of monitoring and things the attenuation coefficient of quality. $\delta$ is random number meeting normal 
distribution, which shows the reality of various interference on the influence of the perceived probability;

(2) All sensor nodes have the ability of data fusion, underwater communications have enough energy to finish self-positioning and can move to the specified location freely;

(3) Before the deployment algorithm execute, all nodes have finished self-positioning accurately, and the nodes' coordinates are known;

(4) There are three kinds of working state in node life: dormancy, detection, communication. The node energy consumption in communication state is the largest, and is smallest in a dormant state. The relationship of energy consumption and the time of nodes in communication state are positive. The larger of communication distance, the more energy is consumed.

DSmT (Dezert - Smarandache found) is put forward by the French scholar Dezert in 2002 [14], and later is developed jointly by Dezert and Smarandache scholar and so on. DSmT is an extension of the classical theory of evidence, but different from basically D-S theory. DSmT can combine with any type of trust function of independent source, but mainly concentrate in the combined uncertainty, high conflict, and inaccurate source of evidence. Especially when the conflict between source become bigger or element is vague, relatively imprecise, DSmT can beyond the limitations of D-S theoretical framework to solve complex fusion problem of static or dynamic [15-20]. definition:

On the basis of the above assumptions and DSmT related theory, we give the following

Definition 1: $p$-reliability coverage: Assume that point $Q$ exists in the monitoring area $H$, in sensor network the credibility of the target node point $Q$ test results $P(Q)$ meet:

$$
P(Q) \geq p(0<p \leq 1)
$$

Definition 2: Effective coverage proportion: If the area $\mathrm{H}$ needed to be monitored is two-dimension area, the acreage of $\mathrm{H}$ is $\mathrm{S}(\mathrm{H})$, the acreage meeting p-reliability coverage is $S_{p}(\mathrm{H})$, and the ratio of $S_{p}(\mathrm{H})$ and $\mathrm{S}(\mathrm{H})$ is:

$$
\eta=\frac{S_{p}(\mathrm{H})}{S(\mathrm{H})} \times 100 \%
$$

Where $\eta$ is the effective coverage proportion of two-dimension monitoring area $\mathrm{H}$.

If the monitoring area by three-dimension area $H$, its volume is $V(H)$, the volume meeting p-reliability coverage is $V_{p}(\mathrm{H})$, and the ratio of $V_{p}(\mathrm{H})$ and $V(\mathrm{H})$ is:

$$
\eta=\frac{V_{p}(\mathrm{H})}{V(\mathrm{H})} \times 100 \%
$$

Where $\eta$ is the effective coverage proportion of 3-D monitoring area $\mathrm{H}$.

Definition 3: Effective detection rate: If there are $N(\mathrm{H})$ target nodes in the monitoring area $\mathrm{H}$, in which $N_{A}(\mathrm{H})$ target nodes are effectively monitored by sensor network, then:

$$
\varphi=\frac{N_{A}(\mathrm{H})}{N(\mathrm{H})} \times 100 \%
$$

Where $\varphi$ is sensor network for monitoring area $\mathrm{H}$ effective detection rate.

Definition 4: Free DSmT model: Set $U$ a recognition framework, $U=\left\{\theta_{1}, \theta_{2}, \ldots, \theta_{n}\right\}$ consist of $n$ detailed elements set (the element in the set can overlap), and other assumptions and constraints of the element (or thesis) are not considered, and then the model of the DSmT model $M^{f}(\mathrm{U})$ is freedom model.

Give a general recognition framework $U$, define a basic probability assignment function $\mathrm{m}: D^{U} \rightarrow[0,1]$ is associated with a given source of evidence, namely: 


$$
m(\varnothing)=0, \sum_{A \in D^{U}} \mathrm{~m}(\mathrm{~A})=1
$$

Where $m(A)$ is generalized the basic probability assignment function of $A$, its trust function and likelihood function is as follows:

$$
\begin{aligned}
& B E L(A)=\sum_{\substack{B \subseteq A \\
B \in D^{U}}} m(B) \\
& P L(A)=\sum_{\substack{B \cap A \neq \varnothing \\
B \in D^{U}}} m(B)
\end{aligned}
$$

When freedom DSmT model work, we have:

$$
\forall A \neq \varnothing \in D^{U}, P L(A)=1
$$

When $n \geq 2$ in Equation (6), the data of each perception atomic which combined into signal source is individual source, mixed DSmT under the mixed free DSmT model of combination rules are as follows:

$$
\begin{aligned}
& \forall A \in D^{U}, m_{M(U)} \stackrel{\text { def }}{=} \varnothing(A)\left[S_{1}(A)+S_{2}(A)+S_{3}(A)\right] \\
& S_{1}(A) \equiv m_{M^{f}(U)}(A) \stackrel{\text { def }}{=} \sum_{\substack{X_{1}, X_{2}, \cdots, X_{n} \in D^{U} \\
X_{1} \cap X_{2} \cap \cdots \cap X_{n}=A}} \prod_{i=1}^{n} m_{i}\left(X_{i}\right)
\end{aligned}
$$

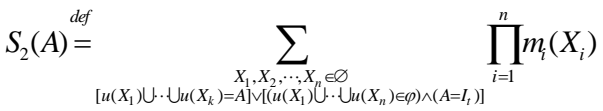

$$
\begin{aligned}
& S_{3}(A)=\sum_{\substack{\text { def } \\
X_{1}, X_{2}, \cdots, X_{n} \in D^{U} \\
\left(X_{1} \cup X_{2} \cup \cdots \cup X_{n}\right)=A \\
X_{1} \cap X_{2} \cap \cdots \cap X_{n} \in \varnothing}} \prod_{i=1}^{n} m_{i}\left(X_{i}\right)
\end{aligned}
$$

\section{Perceptual Element Model}

Organic small molecules is the organic compound whose molecular weight is under 1000 , such as methane $(\mathrm{CH} 4)$, ethane $(\mathrm{C} 2 \mathrm{H} 6)$, ethanol $(\mathrm{C} 2 \mathrm{H} 5 \mathrm{OH})$, benzene $(\mathrm{C} 6 \mathrm{H} 6)$, etc.

The main atoms of small organic molecules are carbon atoms, hydrogen atoms, oxygen atoms, or nitrogen atoms et al. They grouped together according to certain structure is shown in Figure 1, Inspired by this, we combined different structure sensor nodes into one sensing unit according to certain rules. The compound nodes having different complementary sensing nodes can effectively expand the scope of perception, improve the efficiency of a single node's perception, increase effective coverage of sensor networks by appropriate data fusion model.
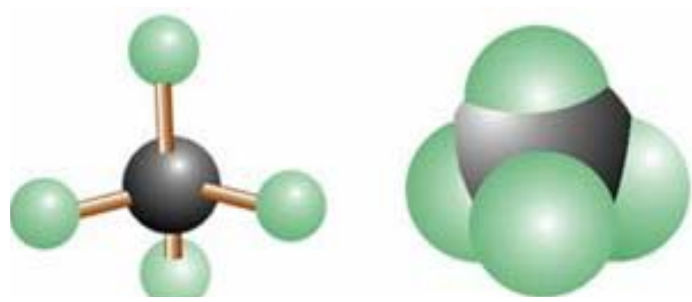

Figure 1. The structure model of methane 
Definition 5: Perceive atoms: Assuming there are $\mathrm{N}$ sensor nodes in the monitoring area $\mathrm{H}$, including $\mathrm{M}$ nodes which have ability to perceiving target node alone, and we call the nodes that can monitor the target and transmit data to the cluster head nodes individually perception atoms.

Definition 6: Perception molecules: Assumed that $\mathrm{n}$ kinds of sensor nodes are deployed in monitoring area, and the different kinds of sensor nodes are combined into sensing units in accordance with the appropriate data fusion model. We call the sensing unit perception molecules. Perception molecules are the smallest perception unit and communication unit.

Assumed that there are $\mathrm{n}$ perception atoms in the molecule, each perception atomic data is $\theta_{1}, \theta_{2}, \cdots, \theta_{\mathrm{n}}$ respectively, and then perception molecular fusion data source is:

$$
\mathrm{U}=\left\{\theta_{1}, \theta_{2}, \ldots, \theta_{n}\right\}
$$

\section{Node Deployment Strategy Based on Organic Small Molecular Model}

With consideration of the perceiving unit model, and data fusion model, we put forward a sensor network node deployment algorithm based on small molecule model as follows:

Step 1: Build appropriate perception model, determine the unit (perception molecule) perception range and the position of the atom in every perception unit according to the monitoring area of $\mathrm{H}$ monitoring requirements. Compute the optimal distance between molecules, and the number of all kinds of sensing nodes.

Step 2: Randomly deploy sensing nodes in the monitoring area $\mathrm{H}$ according to the number of all kinds of sensing nodes in step 1.

Step 3: Select a starting node, which usually is a nodes on vertex positions of monitoring area $\mathrm{H}$, then select the corresponding node closest to the starting node in order to build a sensor molecules, and then its ID is set to 1 .

Step 4: Choose a perception node closest to perception molecules 1 , and build the second perception molecule according to step 3, its ID is set to 2. According to "molecular force" principle, the force between molecules is associated with the between the molecules. Assume that the distance between the two perception molecules is $D^{\prime}$, the best distance for molecules is $D_{i}, \mathrm{t}$ is the threshold value;

When the distance $D^{\prime}$ between perceptions molecules meet:

$$
D^{\prime}<D_{i}-t
$$

The intermolecular force is repulsion, node 2 move far away a units.

When the distance $D^{\prime}$ between perceptions molecules meet:

$$
D^{\prime}>D_{i}+t
$$

The intermolecular force is attraction, node 2 move closely a units.

Until the perception molecular distance meet:

$$
D_{i}-t \leq D^{\prime} \leq D_{i}+t
$$

Node 2 moves completely.

Step 5: By the greedy algorithm, repeat steps 3 and 4, adjust the molecular position, until the entire molecular are adjusted.

Step 6: Each perception molecules complete initialization for data fusion task allocation and data fusion node rotation sequence, and the perception molecular sensors nodes act as data fusion nodes and communication nodes in turn.

Step 7: The data fusion nodes collect the data of other sensors for data fusion at regular intervals.

Step 8: The nodes for data fusion send the data to sink nodes, and then specify a data fusion node and broadcast the messages to other nodes according to the data fusion node rotation order.

TELKOMNIKA Vol. 16, No. 3, December 2015 : $574-582$ 
Step 9: Sensors nodes repeat step 7 and step 8 after receiving the data fusion command.

Pseudo code of the algorithm is as follows:

Function_node_initi(N,1): Initialise the molecule node whose ID is $N$, turn the flag of the node to 1 , determine rotation order of atom nodes that fuse data.

Function_find_min $(N, 0)$ :Find the nearest sensing node $(N+1)$ whose flag is 0, turn the ID to $\mathrm{N}+$ !;

Function_find_min $(N, n)$ :Find $n$ nearest sensing nodes whose flag is 0 , turn the ID to $N+$;

Function_build_molecule $(N)$ :Build the nodes whose ID are $N$ into molecule node, turn the ID to $N$, turn the flag to 1 ;

Function_move_far(N):Move the molecule node $N$ far away from the molecule node $N$ 1;

Function_move_close $(N)$ :Move the molecule node $N$ close to the molecule node $N-1$;

Opt_dist: The optimal distance between two molecule nodes;

Thr_dist; The threshold distance value of the optimal distance.

1. Based on influencing factors, divide diction area to I parts, calculate the number of molecule nodes(Ni), the redundancy rate(Rr) and the optimal distance between to nodes in part I;

2. Deploy $N$ nodes randomly;

3. $N^{\prime}=N^{*} R r$;

4. Wake $N$ ' nodes randomly, flag =0;

5. The flag of node $0=1$;

6. for $(i=0 ; i<N i ; i++)$

7. Function_find_min $(i, 0)$;

8. Function_find_min(i);

9. Function_build_molecule $(i+1)$;

10. if(Dist $>$ Opt_dist+Thr_dist)

11. Function_move_close $(i+1)$;

12. elseif(Dist $<$ Opt_dist-Thr_dist)

13. Function_move_far $(i+1)$;

$14 . \quad$ endif

15. endif

16. endfor

\section{Simulation Analysis}

We simulate NHOS by MATLAB, and assume that the monitoring area $\mathrm{H}$ is square whose side length is $a=3000 \mathrm{~m}$. The aim is to obtain p-reliability coverage in the monitoring area, and $p=0.85$. The effect of target distribution and other environmental factors are not considered.

We assume that two types sensors are used in underwater sensor networks: passive sonar sensor and the giant magnetic resistance sensor. For passive sonar the effective radius of perception is $R_{\text {effic }}=50 \mathrm{~m}$ under the premise of probability coverage.

According to the character of giant magneto resistance sensor and magnetic properties of the target node, the perception range of giant magneto resistance sensor is irregular, and can be assumed to be elliptic, elliptical long radius $R_{a}=60 \mathrm{~m}$ and short radius $R_{b}=30 \mathrm{~m}$ a under the condition of meeting the probability coverage. The numbers of two kinds of sensor are deployed by ratio of 1:3.

Random deployment algorithm and based on the 'virtual force' deployment algorithm simulation respectively. These two kinds of algorithm simulation results compared with the performance of the deployment algorithm based NHOS. The higher of the network coverage rate and the greater effective detection rate of the target node shows that the better performance of network monitoring.

The results are respectively shown in Figure 2, Figure 3 and Figure 4. 


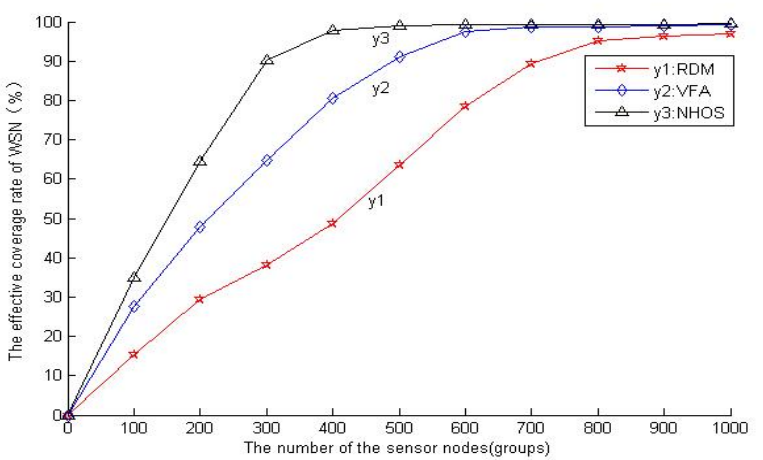

Figure 2. Relations of effective coverage and perceive nodes number

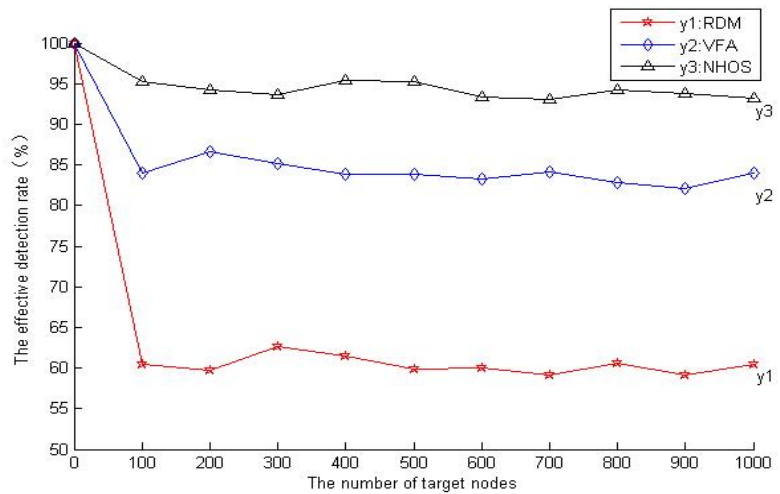

Figure 3. Relations of effective detection rate and target nodes number

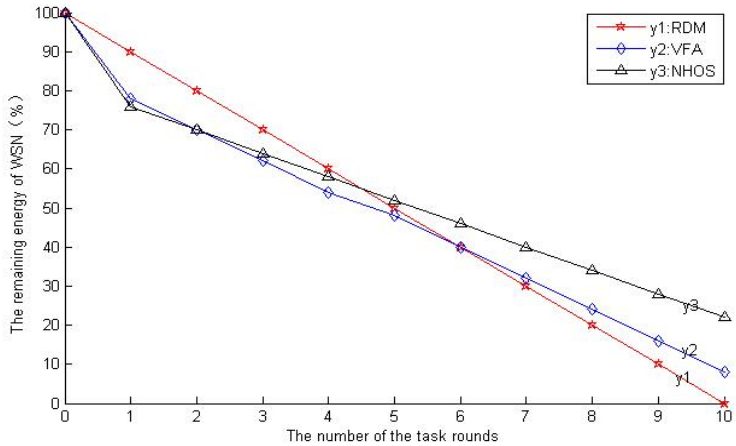

Figure 4. Relations of network residual energy

Figure 2 is shown that the change of the effective coverage rate (p-probability coverage) in the same test area with the increase of the number of sensor node by different algorithms of the RDM, VFA and NHOS algorithm. Compared with RDM algorithm and VFA, NHOS algorithm has more effective coverage and single node's perception is more efficient. $\mathrm{NHOS}$ can reduce the perception scope of sensor node overlapping and the perceived blind area by using DSmT data fusion algorithm. Therefore, NHOS algorithm outperforms VFA algorithm and RDM algorithm in terms of coverage efficiency.

Figure 3 is shown the relationship between effective detection rate and the number of target nodes. As can be seen from the figure 3 that effective detection rate of the target is

TELKOMNIKA Vol. 16, No. 3, December 2015: $574-582$ 
higher in NHOS than in RDM and VFA. The reason is that NHOS adopted DSmT data fusion algorithm which is more effective and can improve the detection probability of the target node, increase the coverage efficiency.

Figure 4 is shown that the change of the node residual energy of sensor networks with the time gone at the same detection area and the same number (500 groups), and the same coverage performance, perception ability of node. As can be seen from the figure 4 that NHOS cost more energy at beginning because of the nodes' movement. VFA algorithm and NHOS cost less energy per unit time than RDM algorithm due to the redundancy node dormancy mechanism of VFA and NHOS. NHOS algorithm consumes less energy than the VFA algorithm. Therefore, NHOS algorithm survives more time compared with other two algorithms.

It can be seen that NHOS can effectively reduce the number of sensor nodes used in the heterogeneous sensor network with the same perception ability, improve the perception efficiency of node, reduce the perception of blind area, and expand the detection range based on the organic small molecular model of heterogeneous sensor network.

\section{Conclusion and Future Work}

In this paper, a heterogeneous sensor network node deployment algorithm NHOS is presented based on the organic small molecular model. DSmT data fusion is used in NHOS to reduce the deployment node number, improve the efficiency of network coverage. We describe the details of our algorithm and compare it with other proposed schemes. Comparison and theory analysis show that the proposed scheme outperforms most of the existing schemes. The further work is to improve, optimize the model in this paper and try to other fusion strategy to optimize the heterogeneous sensor network node deployment algorithm make it more efficient.

\section{Acknowledgements}

The financial supports from the National Natural Science Foundation of China (NSFC) under the grant No.60904023, No.61040010, No.61101167 and the Aeronautical Science Foundation of China (ASFC) under the grant No.20110142003, No.20115142005 are acknowledged gratefully. The authors also gratefully acknowledge the helpful comments and suggestions of the editors and reviewers, which have improved the presentation.

\section{References}

[1] David Moreno-Salinas, Antonio Pascoal, Joaquin Aranda. Sensor Networks for Optimal Target Localization with Bearings-Only Measurements in Constrained Three-Dimensional Scenarios. Sensors. 2013; 13: 10386-10417.

[2] Marjan Moradi, Javad Rezazadeh, Abdul Samad Ismail. A Reverse Localization Scheme for Underwater Acoustic Sensor Networks. Sensors. 2012; 12: 4352-4380.

[3] Achint Aggarwal, Frank Kirchner. Object Recognition and Localization: The Role of Tactile Sensors. Sensors. 2014; 14: 3227-3266.

[4] Luo Xu, Chai Li, Yang Jun. Multi-objective Strategy of Multiple Coverage in Heterogeneous Sensor Networks. Journal of Electronics and Information Technology. 2014; 36(3): 692-695.

[5] Du Xiao-yu, Sun Li-juan, Guo Jian, Han Chong. Coverage Optimization Algorithm for Heterogeneous WSNs. Journal of Electronics and Information Technology. 2014; 36(3): 696-702.

[6] Huang Shuai, Cheng Liang-lun. A Low Redundancy Coverage-enhancing Algorithm for Directional Sensor Network Based on Fictitious Force. Chinese Journal of Sensors and Actuators. 2011; 24(3): 418-422.

[7] Hong Zhen, Yu Li, Zhang Gui-jun. Efficient and Dynamic Clustering Scheme for Heterogeneous Multilevel Wireless Sensor Networks. Acta Automatica Sinica. 2013; 39(4): 454-464.

[8] Li Ming. Study on Coverage Algorithms for Heterogeneous Wireless Sensor Networks. Ph.D. dissertation. Chongqing University; 2011.

[9] Kumar D, Aseri TC, Patel RB. EEHC: Energy Efficient Heterogeneous Clustered Scheme for Wireless Sensor Networks. Computer Communications. 2009; 32(4): 662-667.

[10] Sengupta S, Das S, Nasir MD, et al. Multi-objective Node Deployment in WSNs: in Search of an Optimal Trade-off among Coverage, Lifetime, Energy Consumption, and Connectivity. Engineering Applications of Artificial Intelligence. 2013; 26(1): 405-416.

[11] Bara'a A Attea, Enan A Khalil. A New Evolutionary Based Routing Protocol for Clustered Heterogeneous Wireless Sensor Networks. Apploed Soft Computing. 2012; 12: 1950-1957. 
[12] Hong Zhen, Yu Li, Zhang Gui-jun. Efficient and Dynamic Clustering Scheme for Heterogeneous Multilevel Wireless Sensor Networks. Acta Automatica Sinica. 2013; 39(4): 454-460.

[13] Subir Halder, Sipra Das Bit. Enhancement of Wireless Sensor Network Lifetime by Deploying Heterogeneous Nodes. Journal of Network and Computer Application. 2014; 38: 106-124.

[14] Chen Jie, Du Qing-wei, Li Xiao-yu, Ding Feng. Research on the Deployment Algorithm of Heterogeneous Sensor Networks Based on Probability Model. Journal of Chinese Computer Systems. 2012; 33(1): 50-53.

[15] Cardei M, Thai MT, Ying-shu L, et al. Energy-efficient Target Coverage in Wireless Sensor Networks. 24th Annual Joint Conference of the IEEE Computer and Communications Societies (INFOCOM 2005). Miami. 2005: 1976-1984.

[16] Kashi SS, Sharifi M. Coverage Rate Calculation in Wireless Sensor Networks. Computing. 2012; 94(11): 833-856.

[17] Dezert J. Foundations of a New Theory of Plausible and Paradoxical Reasoning. Information and Security Journal. 2002; 13(9): 90-95.

[18] Dezert J, Tchamov A, Smarandache F, et al. Target Type Tracking with PCR5 and Dempster's Rules: A Comparative Analysis. Proceedings of Fusion 2006 International conference on Information Fusion. Firenze, Italy. 2006.

[19] Smarandache F, Dezert J. Information Fusion Based on New Proportional Conflict Redistribution Rules. Proceedings of Fusion 2005 Conference, Philadelphia, 2005.

[20] Smarandache F, Dezert J. Applications and Advances of DSmT for Information Fusion. Rehoboth: American Research Press. 2006. 